\title{
8 Conclusio
}

Die Integration von Geflüchteten ist in den vergangenen Jahren eines der meistdiskutierten Themen in Politik, Medien und Gesellschaft gewesen. Trotz seiner Bedeutung und häufigen Verwendung ist das Konzept allerdings nicht eindeutig definiert (FASSMANN 2008, p. 4) und daher umstritten. Teilweise wird der Begriff synonym mit Assimilation oder Inklusion gebraucht (vgl. STRASSER 2009, p. 24). Im vorliegenden Forschungsbericht wurde ein potenzialorientierter anstelle eines problemzentrierten Zugangs angewendet, der Integration als Verwirklichungschance (SEN 1999; PERCHING 2010, p. 26) betrachtet. Die Stärke dieses Verständnisses besteht darin, strukturelle Rahmenbedingungen und individuelle Faktoren zu kombinieren und Integration als ein Zusammenspiel beider Aspekte zu betrachten. Im Bereich Bildung - einem zentralen Handlungsfeld von Integration (vgl. FASSMANN o.J.) - wird die Grundlage beispielsweise durch die Bundes- und Landespolitik, den Zugang zu staatlichen Bildungsangeboten, die verfügbaren Ressourcen am Schulstandort oder die infrastrukturellen Voraussetzungen an einem Ort gebildet. Diese können sich positiv oder negativ auf den Integrationsprozess einer Person auswirken. Jedoch kann jeder Mensch mit seiner persönlichen Motivation, seinem Engagement, seiner Resilienzfähigkeit und seinen mitgebrachten Potenzialen und Fähigkeiten innerhalb der vorgefundenen Rahmenbedingungen agieren. Diese Betrachtungsweise von Integration betont die Agency, die Geflüchtete trotz aller Einschränkungen und des fluchtbedingten Statusverlusts im Aufnahmeland besitzen können. Im abschließenden Kapitel werden nun einige zentrale Erkenntnisse dieser Publikation zusammengefasst.

Die Feldforschungen für diesen Forschungsbericht wurden im Jahr 2018 in zwei ländlichen Regionen in Niederösterreich durchgeführt. Zum einen in drei Mostviertler Nachbargemeinden in peripherer Lage und zum anderen in St. Andrä-Wördern, einer Marktgemeinde im Einzugsbereich von Wien. Die unterschiedlichen strukturellen Bedingungen, die Geflüchtete in den Untersuchungsregionen vorfinden, sind ein Beispiel für die Heterogenität des ländlichen Raumes wie beispielsweise bei MACHOLD und DAx 2016 beschrieben. In entlegeneren Gebieten ist die Infrastruktur des öffentlichen Personennahverkehrs häufig schlechter ausgebaut oder aufgrund der längeren Distanzen einfach teurer, sodass Bildungsangebote in größeren Städten oft nur schwer erreicht werden können. Während es für Asylwerber/innen in der Gemeinde St. AndräWördern möglich war, Bildungsangebote in Wien und Tulln zu besuchen, gab es in den Erhebungsgemeinden des Mostviertels kaum entsprechende Möglichkeiten. Die halbstündige Bahnfahrt nach St. Pölten ist recht kostenintensiv, weshalb für Geflüchtete fast ausschließlich jene Angebote in Frage kamen, die vor Ort angeboten wurden. Hierbei zeigte sich, dass infrastrukturelle Faktoren die Verwirklichungschancen von Asylwerber/ inne/n und Asylberechtigten deutlich beeinflussten. Besonders im Hinblick auf die langfristige lokale Integration wirken sich mangelnde Perspektiven im Bildungssektor, auf dem Arbeits- und Wohnungsmarkt sowie fehlende politische Konzepte erheblich auf 
die Wohnortentscheidung von Asylberechtigten nach dem Ende des Verfahrens aus (vgl. OHLinger, SchweIger \& VeYHL 2017). Im Erhebungsgebiet des Mostviertels hat sich gezeigt, dass die Geflüchteten, obwohl sie mancherorts durchaus herzlich aufgenommen wurden und bereits Kontakte zur Aufnahmegesellschaft geknüpft hatten, die Gemeinden nicht als dauerhaften Wohnort in Betracht zogen. Für die Verlagerung des Wohnortes in größere Städte spielen aber auch vielfältige persönliche Gründe eine wichtige Rolle.

Integration kann in vielen verschiedenen Bereichen analysiert werden. In der vorliegenden Publikation stand der Bildungssektor im Mittelpunkt des Erkenntnisinteresses. Bildung besitzt hier eine doppelte Funktion: Sie ist sowohl Instrument für als auch Ziel von Integration (vgl. Ager \& Strang 2008; Kleist 2017, p. 27). Obwohl Österreich seit vielen Jahren Geflüchtete und Migrant/inn/en aufnimmt, ist das nationale Bildungssystem von einigen Mechanismen geprägt, die sich negativ auf die Chancengleichheit unterschiedlicher gesellschaftlicher Gruppen auswirken (vgl. OBERwIMMER et al. 2019, pp. 248 ff.; vgl. LASSNIGG 2015). Für viele neu zugewanderte Menschen stellt das Erlernen der Landessprache Deutsch eine beträchtliche Herausforderung dar. In Österreich ermöglicht die Schulpflicht (geflüchteten) Kindern und Jugendlichen im entsprechenden Alter den Zugang zu Bildung. Kinder mit anderen Erstsprachen haben es jedoch deutlich schwerer, dem Unterricht in allen Gegenständen rasch folgen zu können, wenn keine spezifischen Förderprogramme, wie zum Beispiel DaZ-Unterricht, angeboten werden. Darüber hinaus differenziert das österreichische Schulsystem bereits nach vier Jahren in verschiedene Schultypen mit unterschiedlichem Anforderungsprofil und Bildungs- und Arbeitsmarktchancen nach dem Abschluss. In Bezug auf die Integration von Kindern und Jugendlichen mit Fluchterfahrungen haben die Direktor/inn/ en von Volksschulen und Neuen Mittelschulen in den Erhebungsgemeinden die Erfahrungen gemacht, dass die vorhandenen schulischen Kenntnisse recht unterschiedlich sind. Zusätzlich spielen aber auch andere Faktoren für den Bildungserfolg in Österreich eine wichtige Rolle. Dazu zählen beispielsweise das Alter bei der Einschulung, der Stellenwert von Bildung im Elternhaus (vgl. RothweILER \& RUBERG 2011, pp. 10 ff.), die ergänzenden Fördermöglichkeiten und das Engagement am Schulstandort (vgl. Preuss 2018, pp. 47 ff.). Kinder weisen außerdem unterschiedliche (sprachliche) Begabungen auf, können mit belastenden Erlebnissen vor und während der Flucht besser oder schlechter umgehen und knüpfen schneller oder langsamer neue Freundschaften im Umfeld der Schule. Auf einige der genannten Faktoren haben Schulleiter/innen und Lehrkräfte keinen Einfluss. Ihre pädagogische Aufgabe ist es jedoch, die Teilhabe aller Schüler/innen zu ermöglichen und zu fördern (Preuss 2018, p. 11). Die Schulstandorte in den beiden ländlichen Erhebungsregionen wurden in den Jahren seit 2015 jeweils nur von wenigen Schüler/inne/n mit Fluchterfahrung besucht. Trotzdem ergab sich für die Direktor/inn/en und Lehrer/innen die Herausforderung, die dringend benötigte zusätzliche Förderung, vor allem im sprachlichen Bereich, zu gewährleisten. Hier wurde in fast allen Fällen auf engagierte Freiwillige zurückgegriffen. Dabei handelte es sich entweder um pensionierte Lehrer/innen, Senior/inn/en des Ortes oder Lehrer/innen, die in ihren Freistunden diese Aufgaben übernahmen. Die interviewten Direktor/inn/ 
en brachten zum Ausdruck, dass sie sich im Hinblick darauf mehr Unterstützung und eine flexiblere Verwaltung wünschten, die auf die Erfordernisse bei der Einschulung geflüchteter Schüler/innen (auch während des Schuljahres) eingeht. Die Ergebnisse der empirischen Erhebung zeigten, dass das Engagement von Schulleiter/inne/n und des Lehrerkollegiums in diesem Bereich eine wichtige Rolle spielt, um die Teilhabechancen von geflüchteten Kindern und Jugendlichen zu verbessern. Trotz der strukturellen Rahmenbedingungen gibt es hier einen Gestaltungsspielraum, um am Schulstandort pädagogische Ideale wie Inklusion vorzuleben und umzusetzen (vgl. PILz 2016, p. 64).

Für erwachsene Geflüchtete ist der rechtliche Aufenthaltsstatus entscheidend für den Zugang zum formalen Bildungssektor. Während anerkannte Flüchtlinge Deutschkurse und andere Bildungsmaßnahmen über das AMS besuchen können (vgl. GATTERBAUER 2019), sind diese Angebote für Asylwerber/innen zumindest nicht vorgesehen. In den vergangenen Jahren hat sich gezeigt, dass die Hürden für Asylwerber/innen im Bildungsbereich mit Hilfe von engagierten Freiwilligen durchaus überwunden werden können. Dies kann entweder durch das Angebot eigener kostenloser Bildungsmaßnahmen, wie beispielsweise von Deutschkursen, oder durch Vermittlung und Unterstützung in diesem Bereich, geschehen. Weiters haben niederschwellige Bildungsangebote, wie etwa Basisbildungs- und Pflichtschulabschlusskurse, einen gestiegenen Bedarf seitens der Asylwerber/inne/n und Asylberechtigten abgedeckt, obwohl diese Angebote ursprünglich für andere Zielgruppen konzipiert wurden (für Basisbildung vgl. Kastner 2016, p. 42). So wie Integration weitgehend nicht als einseitiger Prozess der Anpassung der Zuwander/er/innen verstanden wird, sind auch strukturelle Reformen im Bildungssystem von Seiten der Einwanderungsgesellschaft angebracht, um auf die geänderten gesellschaftlichen Rahmenbedingungen einzugehen. Schließlich geht es auch darum, Bildungschancen nicht nur von zivilgesellschaftlichem Engagement und der Unterstützung einzelner Ehrenamtlicher abhängig zu machen, sondern Bildung als Recht zu begreifen, das eng mit den Möglichkeiten gesellschaftlicher Partizipation und Persönlichkeitsentwicklung verbunden ist (vgl. KLOUBERT 2020, p. 3). Im Sinne von Integration als Verwirklichungschance sind Opportunitätsstrukturen im Bildungssektor daher essenziell.

Nicht zuletzt stellt sich im Bildungsbereich die Frage, inwieweit sich die Ausgangslage von Migrant/inn/en und Geflüchteten vergleichen lässt. Für den Ansatz Flucht als Teil von Migration allgemein zu betrachten spricht, dass meist ähnliche Herausforderungen, wie etwa der Spracherwerb, gemeistert werden müssen. Es wird davon ausgegangen, dass diesbezügliche Ergebnisse der allgemeinen Migrationsforschung auf die Situation von Geflüchteten übertragbar sind (EL-MAFAALANI \& MASSUMI 2019, pp. 4 ff.). Allerdings scheint die Perspektive von Flucht als Lebensereignis, das mit spezifischen Herausforderungen und Problematiken verbunden ist, ebenso berechtigt. In diesem Zusammenhang werden häufig psychische Belastungen und Traumata sowie unterbrochene Bildungsbiografien genannt. Aber auch beengte Wohnverhältnisse in Gemeinschaftsunterkünften, die keine geeignete Lernumgebung darstellen, sind häufig Realität von geflüchteten Menschen (El-MAFAalani \& MASSumi 2019, p. 5; DASCHNER 
2017, pp. 16 ff.). Darüber hinaus spielt der rechtliche Aufenthaltsstatus eine große Rolle. Hierbei ist vor allem die Situation von asylwerbenden Familien und deren Kindern zu nennen, die von besonders ausgeprägter Unsicherheit gekennzeichnet ist (vgl. STEWART 2005). Manche waren seit ihrer Ankunft in Österreich bereits mehrfach gezwungen den Wohnort und die Schule zu wechseln, beispielsweise wenn sie in andere Quartiere verlegt worden waren. Dabei darf nicht vergessen werden, dass Geflüchtete keine homogene Gruppe darstellen (vgl. von Dewitz \& Massumi 2017, pp. 29 f.; Preuss 2018, pp. 27 f.; DASCHNER 2017, p. 16). Die Bildungserfahrungen sowie andere individuelle Faktoren können sehr stark variieren. Bei der Ausgestaltung von spezifischen Bildungsmaßnahmen für Geflüchtete sollte außerdem das Alter der Teilnehmer/innen berücksichtigt, sowie für Frauen, die häufig die Betreuung von Kindern übernehmen, Begleitangebote bereitgestellt werden.

Einige Menschen sind darüber hinaus mit formalen Lernangeboten vertraut und können sich beispielsweise im Deutschunterricht recht rasch neue Kenntnisse aneignen. Individuelle Lernstrategien und -präferenzen sind jedoch unterschiedlich, sodass manche Geflüchtete stärker davon profitieren, wenn sie über das Internet oder im Austausch mit anderen Personen, die schon besser Deutsch sprechen, lernen. Insbesondere für Fortschritte in der Umgangssprache ist jedoch die praktische Anwendung im Alltag von zentraler Bedeutung. Für Geflüchtete sind Bildungsangebote, in denen lebensweltliche Inhalte vermittelt werden, von besonderer Wichtigkeit. Ein weiteres Kriterium ist die Rücksichtnahme auf die Lebensumstände von Asylwerber/inne/n und Asylberechtigten in Österreich (ATEş \& REINPRECHT 2017, p. 311). Der kurzfristige Einstieg beziehungsweise Kurskonzepte, die auf einen überschaubaren Zeithorizont angelegt sind, sind hier relevante Charakteristika, die Geflüchtete bei vielen ehrenamtlichen Bildungsangeboten vorfinden und schätzen.

Das Angebot an Bildungsmaßnahmen für Geflüchtete ist in Österreich sehr vielfältig und umfasst neben institutionellen Kursanbietern auch viele Kurse, die von Ehrenamtlichen organisiert wurden und werden. Besonders die Durchführung von Deutschkursen durch engagierte Freiwillige stellt seit dem Jahr 2015 ein zentrales Handlungsfeld zivilgesellschaftlicher Akteur/inn/e/n dar (KaraKaYali \& KLeIST 2015, p. 28). Hierbei ist zu betonen, dass ehrenamtlicher Deutschunterricht für Geflüchtete ganz besondere Qualitäten hat, die professionelle Bildungsangebote kaum leisten können. So ist der persönliche Charakter des Kurssettings hervorzuheben. Im Rahmen des Deutschkurses bauen Unterstützer/innen zu den Asylwerber/inne/n und Asylberechtigten häufig freundschaftliche Beziehungen auf, die über die sozialen Kontakte im Kurs hinausgehen. Engagierte Freiwillige bringen idealerweise die nötige Geduld, Empathie und das Interesse mit, am Alltagsleben der Geflüchteten teilzuhaben. Diese zwischenmenschlichen Beziehungen sind wiederum essenziell für die seelisch-emotionale Integration (HAN-BroICH 2012, pp. 122 ff.) von Asylwerber/inne/n und Asylberechtigten in Österreich. Zudem erweisen sich die Kontakte zu den Ehrenamtlichen als eine wichtige Ressource zum Aufbau von sozialem Kapital. Einige freiwillige Unterstützer/innen betonten, dass ihr Engagement und die damit verbundenen Begegnungen für sie eine Horizonterweiterung und persön- 
liche Bereicherung darstellten und rückten dadurch die Gegenseitigkeit dieser Beziehungen in den Vordergrund. Für manche stand auch der gesamtgesellschaftliche Effekt im Mittelpunkt ihrer Tätigkeit, die in vielen Fällen die Organisation von gemeinsamen Veranstaltungen und individuelle Unterstützung für Geflüchtete miteinschließt. Durch ihr lokales Engagement leisten diese Menschen einen Beitrag für ein harmonisches Zusammenleben im Ort und stärken den sozialen Zusammenhalt (vgl. WAGNER 2019, p. 227). Es ist jedoch wichtig zu betonen, dass diese Angebote von Ehrenamtlichen nicht dazu geeignet sind institutionelle Bildungsmaßnahmen zu ersetzen (vgl. KLEIST 2017, p. 30). Vielmehr geht es darum einen Bedarf abzudecken, dem andernorts nicht ausreichend Beachtung geschenkt wird. Die Verantwortlichkeit für die gelingende Integration von Asylwerber/inne/n und Asylberechtigten auf lokaler und auf nationaler Ebene kann somit nicht auf die Zivilgesellschaft alleine abgeschoben werden, sondern bedarf auch staatlicher Strategien, um längerfristige Erfolge in diesem Bereich sicherzustellen. 